\title{
N-terminal pro-brain natriuretic peptide reflects both left ventricular diastolic dysfunction and myeloma-related renal insufficiency and robustly predicts mortality in patients with symptomatic multiple myeloma
}

\author{
Yoshiaki Abe ${ }^{1, *}$, Tetsuya Kobayashi' ${ }^{2,}{ }^{,}$Yoshiaki Usui ${ }^{3}$, Kentaro Narita ${ }^{1}$, Hiroki \\ Kobayashi $^{1}$, Akihiro Kitadate ${ }^{1}$, Daisuke Miura ${ }^{1}$, Masami Takeuchi ${ }^{1}$ and Kosei \\ Matsue $^{1}$ \\ ${ }^{1}$ Division of Hematology/Oncology, Department of Internal Medicine, Kameda Medical Center, Chiba, Japan \\ ${ }^{2}$ Division of Cardiology, Kameda Medical Center, Chiba, Japan \\ ${ }^{3}$ Division of Cancer Information and Control, Department of Preventive Medicine, Aichi Cancer Centre Research Institute, \\ Aichi, Japan \\ *These authors contributed equally to this work \\ Correspondence to: Yoshiaki Abe, email: yoshiakiabe1018@gmail.com \\ Keywords: diastolic dysfunction; frailty; multiple myeloma; N-terminal pro-brain natriuretic peptide; prognosis \\ Received: October 30, $2018 \quad$ Accepted: January 21, 2019 Published: February 01, 2019 \\ Copyright: Abe et al. This is an open-access article distributed under the terms of the Creative Commons Attribution License 3.0 \\ (CC BY 3.0), which permits unrestricted use, distribution, and reproduction in any medium, provided the original author and source \\ are credited.
}

\section{ABSTRACT}

We retrospectively explored the prognostic relevance of $\mathbf{N}$-terminal pro-brain natriuretic peptide (NT-proBNP) and the association of NT-proBNP with cardiac and renal functions in 153 patients with newly diagnosed symptomatic multiple myeloma and no concomitant light chain amyloidosis who received novel agents. We also examined the usefulness of the new frailty system recently introduced by Mayo Clinic (combining age, performance status, and NT-proBNP). Patients with higher NT-proBNP levels ( $\geq 300 \mathrm{ng} / \mathrm{L}$ ) had a significantly higher incidence of left ventricular diastolic dysfunction (LVDD) and myeloma-related renal insufficiency and significantly shorter overall survival (OS) than did those with lower NT-proBNP levels ( $<300 \mathrm{ng} / \mathrm{L}$ ). NTproBNP remained predictive of OS on multivariate analysis. Mayo Clinic's new frailty system showed excellent discrimination of OS. Furthermore, the Instrumental Activity of Daily Living (IADL) score (not evaluated in Mayo Clinic's study) predicted OS independently of this system, and a sharper discrimination of OS curves was obtained by the incorporation of IADL into this system. Our findings demonstrated that NT-proBNP levels were associated with both LVDD (as a host risk factor) and myeloma-related renal insufficiency (resulting from the disease aggressiveness) and provided predictive information regarding $O S$ in patients with symptomatic myeloma. Furthermore, we, for the first time, validated Mayo Clinic's new frailty system. Our modification further improved Mayo Clinic's system by newly incorporating the IADL score.

\section{INTRODUCTION}

The clinical outcome in patients with symptomatic multiple myeloma (MM) varies depending on intricate interactions between biological properties of the plasma cell clone and host factors [1]. Several prognostic systems have been developed and validated using clinical trials and real-world cohorts [2-5]. Among them, the International Staging System (ISS) and the revised (R)-ISS are the most representative: both systems predominantly include information associated with tumor burden and biological features of myeloma [3, 4]. Meanwhile, several recent studies have shown that patient frailty had an independent influence on mortality in the context of these prognostic 
systems [6-10]. The International Myeloma Working Group (IMWG) proposed a geriatric assessment including age, Katz Activity of Daily Living (ADL), the Lawton Instrumental ADL (IADL), and Charlson Comorbidity Index $(\mathrm{CCI})$, which significantly predicted mortality and the risk of treatment toxicity [8]. Thus, frailty assessment has gained more attention in MM prognostication.

The N-terminal fragments of the prohormone brain natriuretic peptide (NT-proBNP) are secreted predominantly from the left ventricle in response to high ventricular filling pressures and wall stress [11-13]. In addition, plasma NTproBNP concentrations are strongly influenced by renal function. These well-known facts allowed us to speculate that these biomarkers may capture not only the aggressiveness of myeloma but also the host factors, including frailty and tolerability against chemotherapy, and consequently, effectively predict survival. Milani et al. demonstrated the relationship between NT-proBNP levels and patient frailty, delineated by age, Eastern Cooperative Oncology Group performance status (ECOG-PS), ADL, and CCI, further showing the prognostic implications of NT-proBNP in patients with MM using a cohort from Mayo Clinic [14]. They also proposed a new frailty system (combining age, ECOG-PS, and NT-proBNP) as a useful stratification system for MM. However, their cohort included relatively younger patients (median: 65 years) compared to those observed in real-world settings (median: $>70$ years) [15]. Therefore, validation using an independent cohort including real-world population has been needed. In addition, no study has elucidated what the NT-proBNP levels directly reflect in frail myeloma patients or in patients ultimately with risk for all-cause mortality. NT-proBNP has been demonstrated to reflect cardiac dysfunction in heart failure patients [16, 17]; however, MM patients frequently present renal insufficiency, which is unrelated to cardiac functions, and thus, might possibly undermine the performance of NT-proBNP for the evaluation of cardiac function in this population. In this study, we aimed to elucidate the clinical behaviors and usefulness of NT-proBNP in patients with symptomatic $\mathrm{MM}$ by identifying clinical factors and mechanisms that are reflected by NT-proBNP, which more directly affect mortality, and investigating the applicability of the prognostication using NT-proBNP and Mayo Clinic's new frailty system to our real-world cohort.

\section{RESULTS}

\section{Demographic and baseline characteristics of patients}

Baseline clinical characteristics of patients are shown in Table 1. The median age of the patients was 74.2 (interquartile range [IQR]: 66.7-80.8) years. The median observation period for all patients was 26.4 (IQR: 10.849.1) months. Bortezomib and lenalidomide were used in 148 (96.7\%) and 136 (88.9\%) patients, respectively. The median NT-proBNP levels were 309 (IQR: 126-1061) ng/L. Echocardiographic data for the evaluation of left ventricular (LV) diastolic function was available in 131 (85.6\%) patients (routine work-up for diastolic function evaluation was initiated in 2012 at our institution): these patients had no differences in baseline clinical parameters compared with the remaining patients (Supplementary Table 1). Left ventricular diastolic dysfunction (LVDD) was detected in $49.6 \%$ of evaluable patients.

\section{Clinical characteristics of patients according to NT-proBNP levels: association with LVDD and myeloma-related renal insufficiency}

As previously proposed [14], we divided patients into two groups with lower $(<300 \mathrm{ng} / \mathrm{L})$ and higher $(\geq 300 \mathrm{ng} / \mathrm{L})$ NT-proBNP levels. The clinical characteristics of patients with lower $(n=75)$ or higher $(n=78)$ NT-proBNP levels are summarized in Table 1. Patients with higher NTproBNP levels were significantly older; had worse frailty scores; had a higher prevalence of previous histories of arrhythmia and chronic heart failure; had higher beta 2-microglobulin and lactate dehydrogenase levels; and had lower albumin, estimated glomerular filtration rate (eGFR), and hemoglobin levels. Patients with higher NT-proBNP levels also had higher R-ISS stages. However, there were no significant differences between the two groups regarding sex, prevalence of high-risk cytogenetic abnormality (CA) (del $(17 \mathrm{p}), \mathrm{t}(4 ; 14)$, or $\mathrm{t}(14 ; 16)$ detected by interphase fluorescence in situ hybridization analysis), and treatment, including autologous stem cell transplantation (ASCT).

Patients with higher NT-proBNP levels more frequently had LVDD than patients with lower NT-proBNP levels (67.2\% vs. $32.8 \%$, respectively; $P<0.001)$. Furthermore, all diastolic function-related parameters showed associations with NT-proBNP levels, although the difference in septal e' did not reach statistical significance. However, there was no significant difference in the LV ejection fraction (LVEF) between patients with lower and higher NT-proBNP levels.

We also investigated the association between NTproBNP levels and myeloma-related renal insufficiency. Patients with higher NT-proBNP levels more frequently had light chain cast nephropathy ( $41.0 \%$ vs. $4.0 \%$, respectively; $P<0.001$ ) or hypercalcemia-associated renal insufficiency (17.9\% vs. $1.3 \%$, respectively; $P<0.001)$. Such patients also had significantly higher free light chain (FLC) and corrected calcium levels compared to patients with lower NT-proBNP levels. Accordingly, patients with higher NTproBNP levels more frequently had myeloma-related renal insufficiency ( $47.4 \%$ vs. $5.3 \%$, respectively; $P<0.001)$.

\section{Survival outcomes according to the NT-proBNP levels}

Kaplan-Meier survival curves for progressionfree survival (PFS) and overall survival (OS) according 
Table 1: Baseline clinical characteristics of all patients and comparison between patients with lower and higher NT-proBNP levels

\begin{tabular}{|c|c|c|c|c|}
\hline \multirow[b]{2}{*}{ Clinical factors } & \multirow{2}{*}{$\begin{array}{l}\text { All patients } \\
n=153\end{array}$} & \multicolumn{2}{|c|}{ NT-proBNP levels } & \multirow[b]{2}{*}{$\boldsymbol{P}$} \\
\hline & & $\begin{array}{c}\text { Lower } \\
(<300 \mathrm{ng} / \mathrm{L}) \\
n=75\end{array}$ & $\begin{array}{c}\text { Higher } \\
(\geq 300 \mathrm{ng} / \mathrm{L}) \\
n=78\end{array}$ & \\
\hline Observation period, months (median [IQR]) & $26.4[10.8,49.1]$ & $32.8[15.4,53.6]$ & $20.4[10.2,42.7]$ & 0.058 \\
\hline \multicolumn{5}{|l|}{ Background and/or frailty parameters } \\
\hline Age, years (median [IQR]) & $74.2[66.7,80.8]$ & $70.7[65.1,78.1]$ & $75.9[70.2,81.2]$ & 0.020 \\
\hline Sex, male $(\%)$ & $76(49.7)$ & $39(52.0)$ & $37(47.4)$ & 0.62 \\
\hline ECOG-PS, $\geq 2(\%)$ & $83(54.2)$ & $31(41.3)$ & $52(66.7)$ & 0.002 \\
\hline IADL, $\leq 4(\%)$ & $72(47.1)$ & $26(34.7)$ & $46(59.0)$ & 0.003 \\
\hline $\mathrm{CCI}, \geq 2(\%)$ & $74(48.4)$ & $22(29.3)$ & $52(66.7)$ & $<0.001$ \\
\hline \multicolumn{5}{|l|}{ Past medical histories } \\
\hline Coronary artery disease $(\%)$ & $11(7.2)$ & $4(5.3)$ & $7(9.0)$ & 0.53 \\
\hline Arrhythmia (\%) & $10(6.5)$ & $1(1.3)$ & $9(11.5)$ & 0.018 \\
\hline Chronic heart failure (\%) & $15(9.8)$ & $1(1.3)$ & $14(17.9)$ & 0.001 \\
\hline Hypertension $(\%)$ & $86(56.2)$ & $38(50.7)$ & $48(61.5)$ & 0.19 \\
\hline Diabetes mellitus (\%) & $34(22.2)$ & $15(20.0)$ & $19(24.4)$ & 0.56 \\
\hline \multicolumn{5}{|l|}{ Myeloma-related factors } \\
\hline Heavy chain type, $\operatorname{IgG}(\%)$ & $79(51.6)$ & $48(64.0)$ & $31(39.7)$ & 0.004 \\
\hline Light chain only myeloma (\%) & $32(20.9)$ & $10(13.3)$ & $22(28.2)$ & 0.029 \\
\hline Albumin, g/dL (median [IQR]) & $3.4[2.8,3.9]$ & $3.7[3.2,4.0]$ & $3.1[2.5,3.6]$ & $<0.001$ \\
\hline Beta 2-microglobulin, mg/L (median [IQR]) & $4.6[2.9,8.3]$ & $3.0[2.3,4.6]$ & $7.7[4.5,13.9]$ & $<0.001$ \\
\hline eGFR, $\mathrm{mL} / \mathrm{min} / 1.73 \mathrm{~m}^{2}($ median $[\mathrm{IQR}])$ & $50.3[27.8,69.0]$ & $64.8[50.3,74.6]$ & $33.9[13.3,50.5]$ & $<0.001$ \\
\hline Hemoglobin, g/dL (median [IQR]) & $9.6[8.4,11.4]$ & $10.2[9.2,12.2]$ & $9.1[7.8,10.2]$ & $<0.001$ \\
\hline Corrected calcium, mg/dL (median [IQR]) & $9.7[9.2,10.4]$ & $9.4[9.1,9.9]$ & $9.9[9.5,11.0]$ & $<0.001$ \\
\hline Hypercalcemia-associated renal insufficiency (\%) & $15(9.8)$ & $1(1.3)$ & $14(17.9)$ & 0.001 \\
\hline Involved FLC, mg/L (median [IQR]) & $541[128,3225]$ & $360[98,813]$ & $2330[197,8450]$ & $<0.001$ \\
\hline Light chain cast nephropathy (\%) & $35(22.9)$ & $3(4.0)$ & $32(41.0)$ & $<0.001$ \\
\hline Myeloma-related renal insufficiency (\%) & $41(26.8)$ & $4(5.3)$ & $37(47.4)$ & $<0.001$ \\
\hline LDH, high (\%) & $41(26.8)$ & $14(18.7)$ & $27(34.6)$ & 0.029 \\
\hline High-risk CA (\%) & $31(20.3)$ & $15(20.0)$ & $16(20.5)$ & 1.0 \\
\hline R-ISS, $\geq$ stage II (\%) & $126(82.4)$ & $52(69.3)$ & $74(94.9)$ & $<0.001$ \\
\hline DS system, stage III (\%) & $93(60.8)$ & $32(42.7)$ & $61(78.2)$ & $<0.001$ \\
\hline Use of new agents (\%) & $153(100)$ & $75(100)$ & $78(100)$ & 1.0 \\
\hline Bortezomib use (\%) & $148(96.7)$ & $73(97.3)$ & $75(96.2)$ & 1.0 \\
\hline Lenalidomide use (\%) & $136(88.9)$ & $69(92.0)$ & $67(85.9)$ & 0.31 \\
\hline ASCT recipients (\%) & $43(28.1)$ & $26(34.7)$ & $17(21.8)$ & 0.11 \\
\hline \multicolumn{5}{|l|}{ Cardiological parameters } \\
\hline NT-proBNP, ng/L (median [IQR]) & $309[126,1061]$ & $126[65,195]$ & $1058[450,2901]$ & $<0.001$ \\
\hline LVEF, \% (median [IQR]) & $70.0[66.2,73.0]$ & $70.0[67.0,72.0]$ & $70.0[65.7,73.2]$ & 0.90 \\
\hline $\operatorname{LVDD}(\%)^{\dagger}$ & $65(49.6)$ & $22(32.8)$ & $43(67.2)$ & $<0.001$ \\
\hline $\mathrm{E} / \mathrm{e}^{\prime}(\operatorname{median}[\mathrm{IQR}])^{\dagger}$ & $12.6[10.0,15.4]$ & $11.3[9.5,12.9]$ & $14.3[12.4,16.6]$ & $<0.001$ \\
\hline LAVI, $\mathrm{mL} / \mathrm{m}^{2}$ (median [IQR]) & $43.7[33.2,51.4]$ & $40.3[30.4,48.5]$ & $47.5[37.4,59.4]$ & 0.001 \\
\hline Septal e', cm/s (median [IQR]) ${ }^{\dagger}$ & $5.7[4.8,7.1]$ & $5.8[5.3,7.1]$ & $5.6[4.3,7.0]$ & 0.20 \\
\hline TR velocity, m/s (median $[\mathrm{IQR}])^{\dagger}$ & $2.60[2.33,2.83]$ & $2.4[2.2,2.6]$ & $2.8[2.6,3.1]$ & $<0.001$ \\
\hline
\end{tabular}

Abbreviations: ASCT, autologous stem cell transplantation; CA, cytogenetic abnormality; CCI, Charlson Comorbidity Index; DS, Durie-Salmon; ECOGPS, Eastern Cooperative Oncology Group performance status; eGFR, estimated glomerular filtration rate; FLC, free light chain; IADL, Instrumental Activity of Daily Living; IQR, interquartile range; LAVI, left atrial volume index; LDH, lactate dehydrogenase; LVDD, left ventricular diastolic dysfunction; LVEF, left ventricular ejection fraction; NT-proBNP, N-terminal pro-brain natriuretic peptide; R-ISS, revised International Staging System; TR, tricuspid regurgitation.

${ }^{\dagger} n=131$ (lower NT-proBNP, $n=67$; higher NT-proBNP, $n=64$ ). 
to NT-proBNP levels are shown in Figure 1. Patients with higher NT-proBNP levels had both significantly shorter PFS and OS than patients with lower NT-proBNP levels (median PFS: 24.6 and 67.1 months, respectively; $P<0.001$, and median OS: 40.4 months and not reached, respectively; $P<0.001)$. Patients with higher NT-proBNP levels had significantly shorter OS than patients with lower NT-proBNP levels in both younger $(<70$ years) or older ( $\geq 70$ years) groups (Supplementary Figure 1$)$ and in both higher $\left(\geq 50 \mathrm{ml} / \mathrm{min} / 1.73 \mathrm{~m}^{2}\right)$ and lower $(<50 \mathrm{ml} /$ $\mathrm{min} / 1.73 \mathrm{~m}^{2}$ ) eGFR groups (Supplementary Figure 2). The NT-proBNP levels also discriminated two groups with different OS among both 43 and 110 patients who received treatment with and without ASCT, respectively (Supplementary Figure 3). Furthermore, there was a sharp discrimination of the OS curves based on the NT-proBNP levels among both patients with R-ISS stage II and stage III (Supplementary Figure 4).

On univariate analysis, age, ECOG-PS, IADL, CCI, NT-proBNP, LVDD, and the R-ISS significantly predicted OS. Despite the relationship between NT-proBNP levels and other clinical factors, NT-proBNP retained its prognostic value for OS in the multivariate analysis (Supplementary Table 2). Notably, LVDD also showed independent prognostic impact for OS.

\section{Validation and modification of Mayo Clinic's new frailty system}

As described in a previous report from Mayo Clinic [14], we divided patients into four groups using Mayo

\section{A PFS}

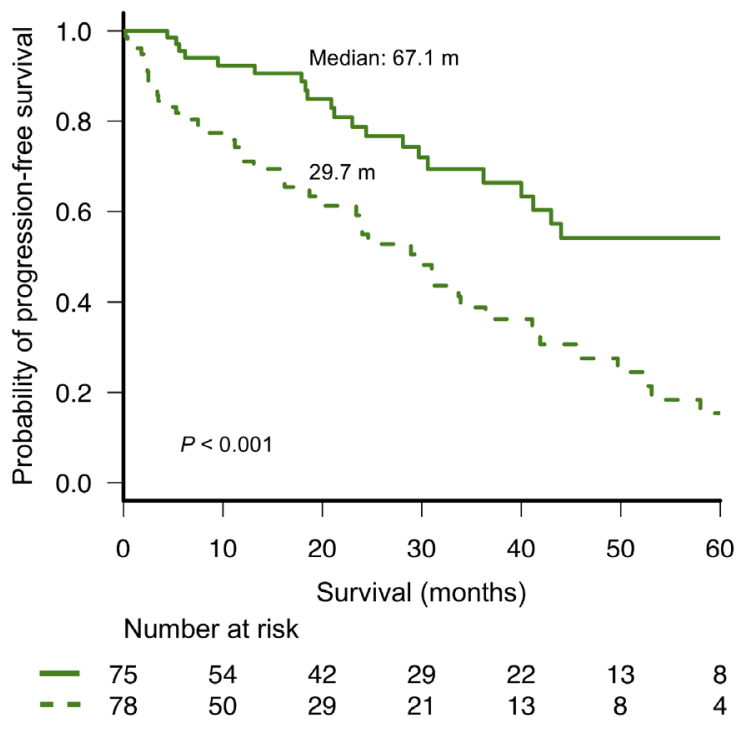

Clinic's new frailty system that incorporated three risk factors: age $\geq 70$ years, ECOG-PS $\geq 2$, and NT-proBNP levels $\geq 300 \mathrm{ng} / \mathrm{L}$. This frailty system sharply discriminated four different groups in the entire cohort as well as in patients with or without high-risk CAs, as observed in Mayo Clinic's previous study (Figure 2). We further analyzed the value of this frailty system by adjusting for the IADL score, which was not evaluated in the previous study conducted by Mayo Clinic. Notably, the IADL was still an independent prognostic parameter for OS even after adjusting for Mayo Clinic's new frailty system and the R-ISS (Table 2A). Therefore, we modified Mayo Clinic's new frailty system by incorporating the IADL score; this modified, new frailty system included age $\geq 70$ years, ECOG-PS $\geq 2$, IADL $\leq 4$, and NT-proBNP levels $\geq 300 \mathrm{ng} / \mathrm{L}$, with each parameter scoring 1 point, thus creating a staging system ranging from I to V. Notably, this modified frailty system showed excellent discrimination of the OS curves across the five groups (Figure 3). Furthermore, this modified frailty system remained an independent predictor of the R-ISS in multivariate analysis (Table 2B). Receiver operating characteristic (ROC) curves were developed for a basic model that consists of the variables used in Mayo Clinic's new frailty system and a model consisting of this basic model + IADL for 5-year OS (Supplementary Figure 5). Incorporation of IADL into the basic Mayo Clinic's frailty model resulted in an increase in area under curve (AUC) from 0.80 (95\% confidence interval $[\mathrm{CI}] ; 0.68-0.92)$ to 0.87 (95\% CI; 0.78-0.96), although it did not reach statistical significance $(P=0.10)$. Calibration analysis showed an agreement between the

\section{B OS \\ - NT-proBNP $<300 \mathrm{ng} / \mathrm{L}$ - - NT-proBNP $\geq 300 \mathrm{ng} / \mathrm{L}$}

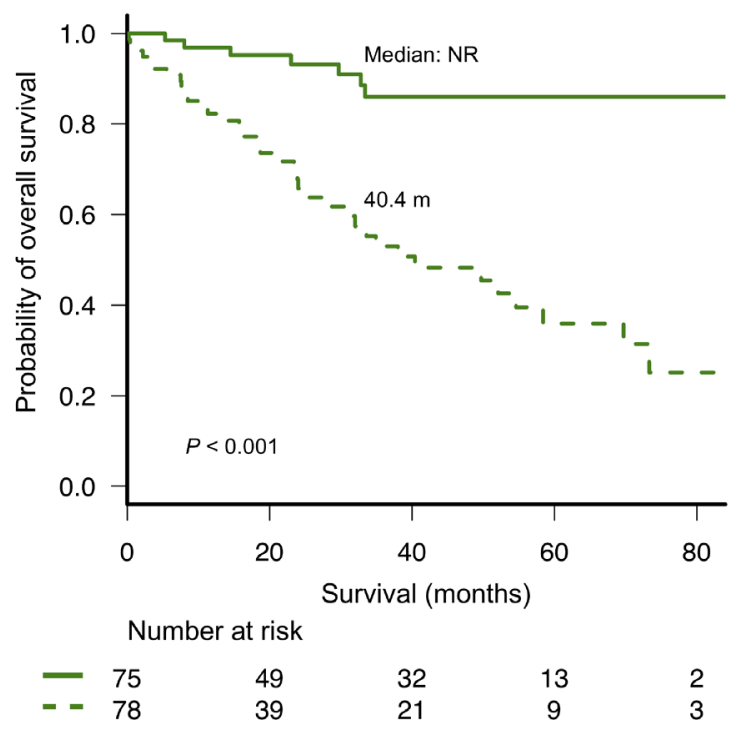

Figure 1: (A) Progression-free survival (PFS) and (B) overall survival (OS) according to the levels ( $<$ or $\geq 300 \mathrm{ng} / \mathrm{L})$ of N-terminal probrain natriuretic peptide (NT-proBNP). 
observed risk and the risk predicted by Mayo Clinic's frailty system model and our modified, new frailty system model ( $P=0.64$ and $P=0.15$, respectively). Continuous net reclassification improvement (NRI) and integrated discrimination improvement (IDI) were significant (NRI 1.093 [95\% CI; 0.564-1.621], $P<0.001$; IDI 0.070 [95\% CI; 0.001-0.138], $P=0.042$ ).

\section{A The entire cohort}

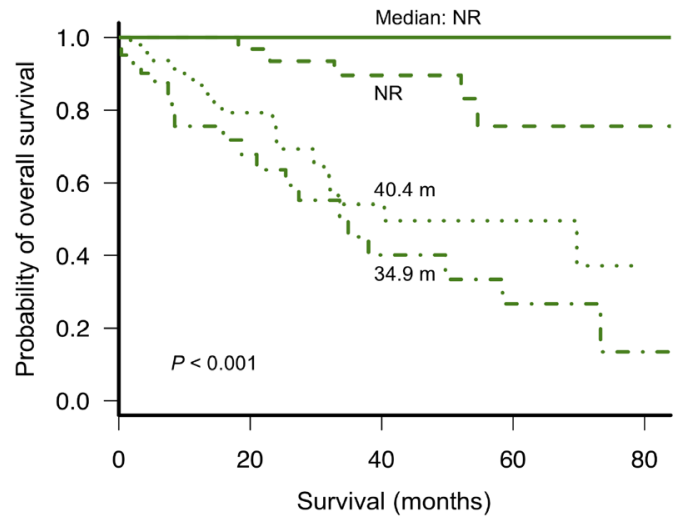

Number at risk

$\begin{array}{cccccc}- & 22 & 16 & 14 & 6 & 2 \\ -- & 42 & 29 & 20 & 8 & 2 \\ \ldots- & 48 & 27 & 12 & 5 & 0 \\ -. & 41 & 16 & 7 & 3 & 1\end{array}$

\section{B Standard risk CAs}

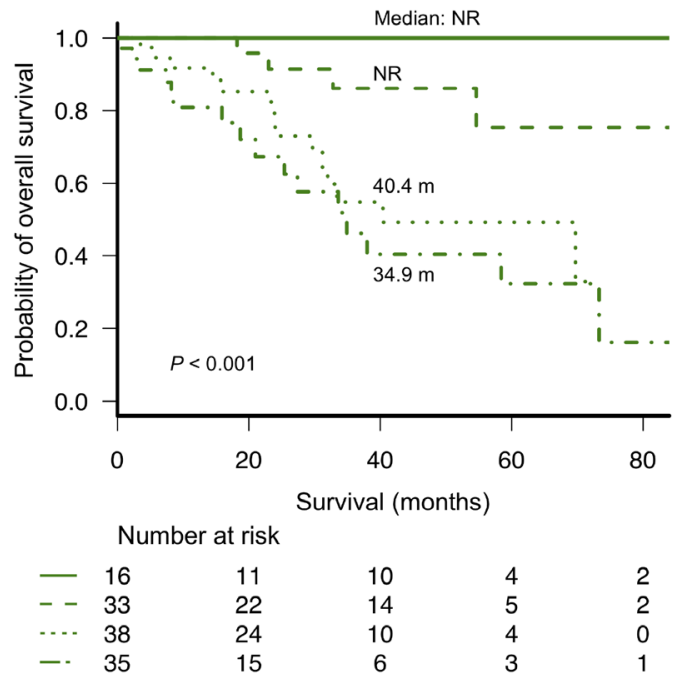

\section{Changes in NT-proBNP levels on follow-up tests according to the presence of LVDD or myeloma- related renal insufficiency}

Figure 4A and 4B show the NT-proBNP levels on pretreatment tests and follow-up tests at best response in patients with only LVDD $(n=17)$ and those with only

$$
\begin{aligned}
& \text { - Stage I } \\
& -- \text { Stage II } \\
& \ldots \text {-.. Stage III } \\
& \text { - } \text { Stage IV }
\end{aligned}
$$

\section{c High-risk CAs}

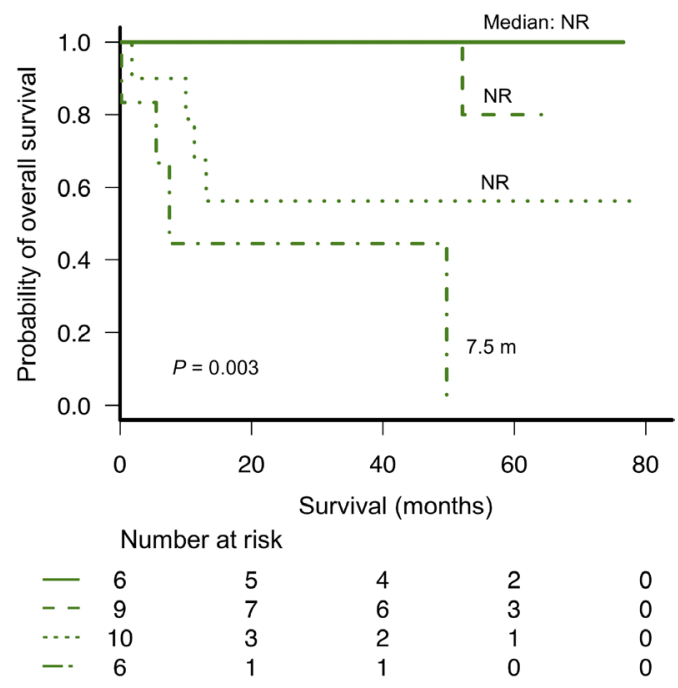

Figure 2: Overall survival according to Mayo Clinic's new frailty system based on age $\geq 70$ years, ECOG-PS $\geq 2$, and NT-proBNP $\geq \mathbf{3 0 0} \mathrm{ng} / \mathrm{L}$ (different stages based on a score of $\mathbf{0}-\mathbf{3}$ points, respectively). (A) overall survival according to the frailty system in the entire cohort, $(\mathbf{B})$ overall survival according to the frailty system in patients without high-risk cytogenetic abnormalities, and (C) overall survival according to the frailty system in patients with high-risk cytogenetic abnormalities [del(17p), $\mathrm{t}(4 ; 14)$, or $\mathrm{t}(14 ; 16)$ detected by fluorescence in situ hybridization analysis]. 
Table 2: Multivariate Cox regression analyses predicting overall survival

\begin{tabular}{lcc}
\hline $\mathbf{A}$ & & \\
\hline Variables & HR $\mathbf{( 9 5 \%} \mathbf{C I})$ & $\boldsymbol{P}$ \\
\hline IADL, $\leq 4$ & $2.25(1.06-4.78)$ & 0.035 \\
CCI, $\geq 2$ & $0.67(0.32-1.40)$ & 0.29 \\
Mayo Clinic's new frailty system (ECOG-PS-Age-NT-proBNP) & $2.04(1.35-3.10)$ & 0.001 \\
R-ISS & $2.00(1.09-3.67)$ & 0.024 \\
\hline B & & $\boldsymbol{P}$ \\
\hline Variables & HR (95\% CI) & $<0.001$ \\
\hline Modified new frailty system (ECOG-PS-IADL-Age-NT-proBNP) & $1.62(1.28-2.06)$ & 0.032 \\
R-ISS & $1.88(1.05-3.36)$ & \\
\hline
\end{tabular}

Abbreviations: CCI, Charlson Comorbidity Index; CI, confidence interval; ECOG-PS, Eastern Cooperative Oncology Group performance status; HR, hazard ratio; IADL, Instrumental Activity of Daily Living; NT-proBNP, N-terminal probrain natriuretic peptide; R-ISS, revised International Staging System.

myeloma-related renal insufficiency $(n=12)$ who had higher NT-proBNP levels on pretreatment tests. With a median time interval of 3.6 (IQR: 2.5-6.4) months, NT-proBNP levels in patients with only LVDD showed no significant change at best response compared with the pretreatment test levels. In $5(29.4 \%)$ of these patients, NT-proBNP levels decreased by half; these patients had no difference in specific clinical characteristics compared to that of the remaining patients. Conversely, NT-proBNP levels in patients with only myeloma-related renal insufficiency showed a significant reduction from the pretreatment levels with a median time interval of 2.6 (IQR: 1.4-3.8) months $(P<0.001)$.

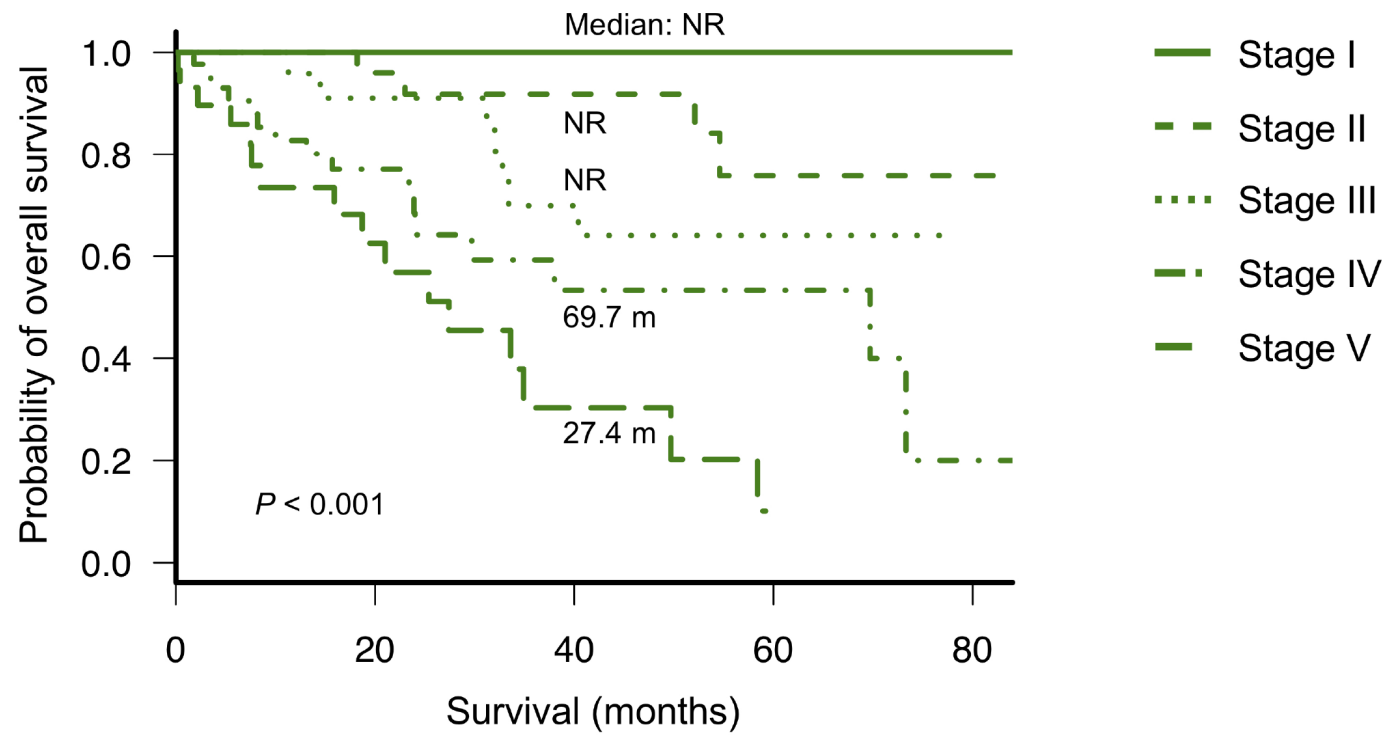

\section{Number at risk}

$\begin{array}{cccccc}- & 21 & 15 & 13 & 6 & 2 \\ -= & 32 & 23 & 16 & 7 & 2 \\ \cdots & 28 & 18 & 12 & 4 & 0 \\ -= & 43 & 21 & 9 & 5 & 1 \\ - & 29 & 11 & 3 & 0 & 0\end{array}$

Figure 3: Overall survival according to the modified new frailty system based on age $\geq 70$ years, ECOG-PS $\geq 2$, IADL $\leq 4$, and NT-proBNP $\geq 300 \mathrm{ng} / \mathrm{L}$ (different stages based on a score of 0 to 4 points). ECOG-PS, Eastern Cooperative Oncology Group performance status; IADL, Instrumental Activity of Daily Living; NT-proBNP, N-terminal pro-brain natriuretic peptide. 


\section{DISCUSSION}

In the present study, we investigated the relationship between NT-proBNP levels and clinical parameters, including frailty scores, cardiac (including LV diastolic function), and renal function, in real-world patients with symptomatic MM with no concomitant light chain (AL) amyloidosis. We further sought to validate the prognostic value of the NT-proBNP levels as well as the new frailty system suggested by the Mayo Clinic study [14].

NT-proBNP has shown robust prognostic relevance in AL amyloidosis [18] and has thus been incorporated into major staging systems for diagnosis [19]. In addition, previous studies in $\mathrm{AL}$ amyloidosis have demonstrated that NT-proBNP levels differ in patients with or without cardiac involvement characterized by ventricular wall thickness. However, its prognostic performance and cardiac disorder patterns shown by NT-proBNP, which may have a negative prognostic impact, have not been fully elucidated in symptomatic MM. The results of this study suggested that NT-proBNP had prognostic significance even in symptomatic MM, especially for OS. These reproducible results observed across different disease entities might be related to the potential capability of NT-proBNP to reflect both cardiac and renal dysfunctions with great sensitivity on the basis of unclarified molecular mechanisms in patients whose hearts and kidneys are concurrently affected.

Our data revealed that NT-proBNP levels were associated with the incidence of LVDD, as previously described in non-cancer-bearing patients [20], but not with conventional LVEF in MM. Furthermore, the presence of LVDD was detected as an independent predictor for OS. The prognostic implications of LVDD may be partly related to its association with tolerability against cardiotoxicity of anti-myeloma agents such as proteasome inhibitors (PIs) and cytotoxic agents including high-dose chemotherapy for ASCT recipients [21, 22]. In particular, PIs remarkably improved survival in MM [23], despite its potential cardiotoxicity [24]. Therefore, novel agent-based treatments might have made the prognostic relevance of subclinical cardiac dysfunctions greater, relative to that in the pre-novel agent era. Additionally, the negative influence of LVDD has been described even in the context of the natural history of healthy subjects [25]. Thus, we believe that the assessment of LV diastolic function will be of utmost importance in the evaluation of host factors for the prognostication of MM patients who are treated with or without PIs.

We also revealed that NT-proBNP was associated with myeloma-related renal insufficiency based on the high frequency of light chain cast nephropathy and hypercalcemia-associated renal insufficiency among patients with higher NT-proBNP levels. Several studies have shown that high levels of baseline serum calcium and involved FLC are associated with disease aggressiveness and unfavorable outcomes in symptomatic myeloma [26-28]. In contrast to LVDD patients, NT-proBNP levels in patients with myeloma-related renal insufficiency significantly decreased at disease remission. Accordingly, NT-proBNP possibly reflects both host and disease factors and could be used as a comprehensive marker for

\section{B Myeloma-related renal insufficiency only}
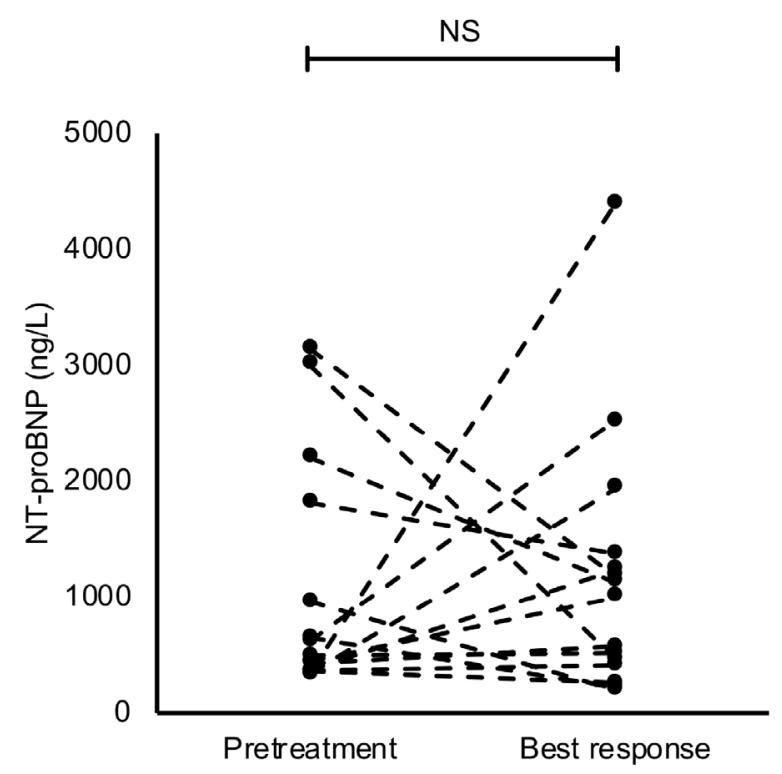
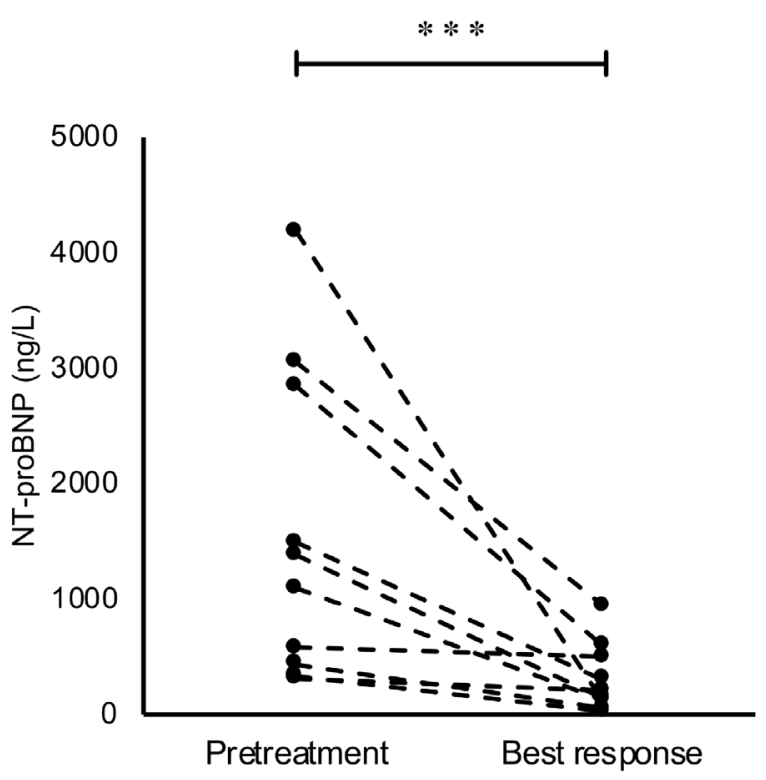

Figure 4: Changes in the levels of N-terminal pro-brain natriuretic peptide (NT-proBNP) on follow-up tests at best response in (A) patients with only left ventricular diastolic dysfunction (LVDD) and (B) patients with only myeloma-related renal insufficiency. Asterisks $\left.{ }^{*}\right)$ indicate statistical significance: ${ }^{* * *} P<0.001$, NS: not significant. 
the prognostic assessment of MM. Indeed, NT-proBNP levels discriminated between patients with significantly different survivals even in both younger and older patients or in patients with or without decreased renal functions. Furthermore, multivariate analysis identified NT-proBNP as an independent prognostic predictor.

In comparison to the cohort in the previous report from Mayo Clinic [14], our cohort included considerably older patients (median age: 65 vs. 74 years) reflecting the real-world Japanese population. As a result, the absolute NT-proBNP levels as well as the frailty scores were greater in our cohort [29]. However, Mayo Clinic's new frailty system demonstrated excellent performance in our cohort despite this clear age difference. These results are of particular interest because the peculiar distribution of patients' age observed in our study may exemplify the future situations in other developed Western countries approaching a period of super-aging such as that currently observed in Japan. Moreover, we found a prognostic value for the IADL score, which was not evaluated in Mayo Clinic's study despite its established utility in the geriatric assessment [8], independent of the new frailty system; the incorporation of the IADL could further improve this frailty system.

The present study has several limitations, including its retrospective nature, heterogeneous treatments, and relatively small sample size. Despite these limitations, this study highlighted the association between NT-proBNP and LV diastolic function or myeloma-related renal insufficiency and a promising prognostic potential of NTproBNP per se or as a part of the newer frailty systems in patients with symptomatic myeloma.

In conclusion, our findings revealed that the baseline NT-proBNP levels were associated with both LVDD (as a host risk factor) and myeloma-related renal insufficiency (resulting from disease aggressiveness). In addition, this is the first validation study of Mayo Clinic's new frailty system. Our results validated the robust prognostic relevance of NT-proBNP and the usefulness of Mayo Clinic's new frailty system in a real-world context. Moreover, our modification further improved Mayo Clinic's system by newly incorporating the IADL score. Further longitudinal studies are warranted to validate our results and establish the value of NT-proBNP and these frailty systems for the routine assessment of symptomatic $\mathrm{MM}$ in the era of novel agents.

\section{MATERIALS AND METHODS}

\section{Study design and patients}

This study retrospectively analyzed data of 153 consecutive newly diagnosed symptomatic MM patients treated with chemotherapy between April 2011 and March 2018 at Kameda Medical Center, Chiba, Japan. We routinely perform bone marrow and subcutaneous adipose tissue biopsy; further, we perform biopsies of targeted organs when suspected for the detection of light chain AL amyloidosis in all patients. Patients with pathologically proven involvement with AL amyloidosis were excluded from the analysis. The diagnoses and treatment responses were evaluated using the IMWG criteria $[30,31]$. We included only patients who had been treated with novel agents (e.g., immunomodulatory agents or PIs). As part of the initial evaluations of patients at admission, ECOGPS, IADL score, and CCI were routinely calculated. Pretreatment levels of NT-proBNP were routinely measured on sera using standard commercially available assays. Serum FLC levels were quantified in all patients with the use of the Freelite assay (The Binding Site, UK) on a Dade-Behring BN II Nephelometer. Serum calcium levels were corrected for hypoalbuminemia appropriately. The presence of LVDD was determined individually according to the 2016 American Society of Echocardiography and European Association of Cardiovascular Imaging guidelines [32], which is briefly described in Doc. S1. The diagnoses of renal insufficiency, light chain cast nephropathy, and hypercalcemia were made according to the updated IMWG criteria [30], which is also briefly described in Doc. S1. Myeloma-related renal insufficiency included light chain cast nephropathy and hypercalcemia-associated renal insufficiency in this study. Written informed consent was obtained from all patients or their families. The study was conducted according to the Declaration of Helsinki and was approved by the review board of Kameda Medical Center.

\section{Statistical analysis}

The relationship between baseline characteristics and NT-proBNP levels were compared using one-way analysis of variance, Kruskal-Wallis tests, Wilcoxon signed rank tests, or chi-squared tests, as appropriate. PFS was defined as the time from the date of diagnosis to the date of confirmation of the first disease progression or death from any cause, whichever occurred first. OS was defined as the time from the diagnosis to the date of death from any cause or last follow-up [4]. The probability of PFS and OS was determined using the Kaplan-Meier method and compared using the log-rank test. The prognostic impact of NT-proBNP levels as well as the frailty systems was evaluated using multivariate Cox proportional-hazards analyses. In the multivariate analyses, adjustment was performed for age, other frailty scores, and R-ISS, as previously described in Mayo Clinic's study [14].

To assess the effect of the addition of IADL data on Mayo Clinic's frailty system, we tested its discrimination and reclassification capabilities by the ROC curve and AUC, NRI, and IDI. We constructed a ROC curve for two logistic regression models. The basic model was constructed with variables which comprise Mayo Clinic's frailty system (age, ECOG-PS, and NT-proBNP), while 
the basic model + IADL model was constructed with the basic model plus IADL. Difference in the AUC was compared using DeLong's approach [33]. Continuous NRI and IDI were also determined to evaluate the additive prognostic value of IADL [34]. The goodness-of-fit test statistics were also calculated to assess the calibration for the prediction models using the Hosmer-Lemeshow method [35]. A two-tailed $P$ value $<0.05$ was considered statistically significant. Statistical analysis was performed using STATA version 15.1 (Stata corp., College Station, TX, USA) for the ROC analyses and R version 3.1.2 (R Foundation for Statistical Computing, Vienna, Austria) for the remaining analyses.

\section{Abbreviations}

ADL: activity of daily living; AL amyloidosis: light chain amyloidosis; AUC: area under the curve; ASCT: autologous stem cell transplantation; CA: cytogenetic abnormality; CCI: Charlson Comorbidity Index; CI: confidence interval; ECOG-PS: Eastern Cooperative Oncology Group performance status; eGFR: estimated glomerular filtration rate; FLC: free light chain; IADL: Instrumental Activity of Daily Living; IMWG: International Myeloma Working Group; IQR: interquartile range; ISS: International Staging System; LV: left ventricular; LVDD: left ventricular diastolic dysfunction; LVEF: left ventricular ejection fraction; MM: multiple myeloma; NT-proBNP: N-terminal pro-brain natriuretic peptide; OS: overall survival; PFS: progression-free survival; PI: proteasome inhibitor; R-ISS: Revised International Staging System; ROC: receiver operating characteristic.

\section{Author contributions}

YA planned, initiated, designed the study, collected data, performed statistical analyses, wrote the manuscript, and provided patient care. TK collected data and provided patient care. YU performed statistical analyses. KN, HK, AK, DM, and MT provided patient care. KM supervised the study, wrote the manuscript, and provided patient care. All authors reviewed and approved the manuscript.

\section{ACKNOWLEDGMENTS}

The authors would like to thank the cardiology department staff at Kameda Medical Center in Kamogawa, Japan, for their help in the acquisition and interpretation of echocardiography images. We also would like to thank Soichi Kurihara for specimen collection and NT-proBNP measurements. Finally, we thank Editage (https://www. editage.jp/) for English language editing.

\section{CONFLICTS OF INTEREST}

The authors have no conflicts of interest.

\section{FUNDING}

None.

\section{REFERENCES}

1. Kumar SK, Rajkumar V, Kyle RA, van Duin M, Sonneveld P, Mateos MV, Gay F, Anderson KC. Multiple myeloma. Nat Rev Dis Primers. 2017; 3:17046. https://doi. org/10.1038/nrdp.2017.46.

2. Durie BG, Salmon SE. A clinical staging system for multiple myeloma. Correlation of measured myeloma cell mass with presenting clinical features, response to treatment, and survival. Cancer. 1975; 36:842-54.

3. Greipp PR, San Miguel J, Durie BG, Crowley JJ, Barlogie B, Blade J, Boccadoro M, Child JA, Avet-Loiseau H, Kyle RA, Lahuerta JJ, Ludwig H, Morgan G, et al. International staging system for multiple myeloma. J Clin Oncol. 2005; 23:3412-20. https://doi.org/10.1200/JCO.2005.04.242.

4. Palumbo A, Avet-Loiseau H, Oliva S, Lokhorst HM, Goldschmidt H, Rosinol L, Richardson P, Caltagirone S, Lahuerta JJ, Facon T, Bringhen S, Gay F, Attal M, et al. Revised International Staging System for Multiple Myeloma: A Report From International Myeloma Working Group. J Clin Oncol. 2015; 33:2863-9. https://doi. org/10.1200/JCO.2015.61.2267.

5. Kastritis E, Terpos E, Roussou M, Gavriatopoulou M, Migkou M, Eleutherakis-Papaiakovou E, Fotiou D, Ziogas D, Panagiotidis I, Kafantari E, Giannouli S, Zomas A, Konstantopoulos K, et al. Evaluation of the Revised International Staging System in an independent cohort of unselected patients with multiple myeloma. Haematologica. 2017; 102:593-9. https://doi.org/10.3324/ haematol.2016.145078.

6. Ludwig H, Durie BG, Bolejack V, Turesson I, Kyle RA, Blade J, Fonseca R, Dimopoulos M, Shimizu K, San Miguel J, Westin J, Harousseau JL, Beksac M, et al. Myeloma in patients younger than age 50 years presents with more favorable features and shows better survival: an analysis of 10549 patients from the International Myeloma Working Group. Blood. 2008; 111:4039-47. https://doi. org/10.1182/blood-2007-03-081018.

7. Ludwig H, Bolejack V, Crowley J, Blade J, Miguel JS, Kyle RA, Rajkumar SV, Shimizu K, Turesson I, Westin J, Sonneveld P, Cavo M, Boccadoro M, et al. Survival and years of life lost in different age cohorts of patients with multiple myeloma. J Clin Oncol. 2010; 28:1599-605. https://doi.org/10.1200/jco.2009.25.2114.

8. Palumbo A, Bringhen S, Mateos MV, Larocca A, Facon T, Kumar SK, Offidani M, McCarthy P, Evangelista A, Lonial S, Zweegman S, Musto P, Terpos E, et al. Geriatric assessment predicts survival and toxicities in elderly myeloma patients: an International Myeloma Working Group report. Blood. 2015; 125:2068-74. https://doi. org/10.1182/blood-2014-12-615187. 
9. Rosko AE, Huang Y, Benson DM, Efebera YA, Hofmeister C, Jaglowski S, Devine S, Bhatt G, Wildes TM, Dyko A, Jones D, Naughton MJ, Byrd JC, et al. Use of a comprehensive frailty assessment to predict morbidity in patients with multiple myeloma undergoing transplant. J Geriatr Oncol. 2018 Jul 5. [Epub ahead of print]. https://doi. org/10.1016/j.jgo.2018.05.015.

10. Wildes TM, Tuchman SA, Klepin HD, Mikhael J, Trinkaus K, Stockerl-Goldstein K, Vij R, Colditz G. Geriatric Assessment in Older Adults with Multiple Myeloma. J Am Geriatr Soc. 2018 Dec 13. [Epub ahead of print]. https://doi. org/10.1111/jgs. 15715 .

11. Kinnunen P, Vuolteenaho O, Ruskoaho H. Mechanisms of atrial and brain natriuretic peptide release from rat ventricular myocardium: effect of stretching. Endocrinology. 1993; 132:1961-70. https://doi.org/10.1210/endo.132.5.8477647.

12. Richards AM, Crozier IG, Yandle TG, Espiner EA, Ikram H, Nicholls MG. Brain natriuretic factor: regional plasma concentrations and correlations with haemodynamic state in cardiac disease. Br Heart J. 1993; 69:414-7.

13. Magga J, Vuolteenaho $\mathrm{O}$, Tokola $\mathrm{H}$, Marttila $\mathrm{M}$, Ruskoaho H. B-type natriuretic peptide: a myocytespecific marker for characterizing load-induced alterations in cardiac gene expression. Ann Med. 1998; 30:39-45.

14. Milani P, Vincent Rajkumar S, Merlini G, Kumar S, Gertz MA, Palladini G, Lacy MQ, Buadi FK, Hayman SR, Leung N, Dingli D, Lust JA, Lin Y, et al. N-terminal fragment of the type-B natriuretic peptide (NT-proBNP) contributes to a simple new frailty score in patients with newly diagnosed multiple myeloma. Am J Hematol. 2016; 91:1129-34. https://doi.org/10.1002/ajh.24532.

15. Blimark $\mathrm{CH}$, Turesson I, Genell A, Ahlberg L, Bjorkstrand B, Carlson K, Forsberg K, Juliusson G, Linder O, Mellqvist UH, Nahi H, Kristinsson SY. Outcome and survival of myeloma patients diagnosed 2008-2015. Real-world data on 4904 patients from the Swedish Myeloma Registry. Haematologica. 2018; 103:506-13. https://doi.org/10.3324/haematol.2017.178103.

16. Tschope C, Kasner M, Westermann D, Gaub R, Poller WC, Schultheiss HP. The role of NT-proBNP in the diagnostics of isolated diastolic dysfunction: correlation with echocardiographic and invasive measurements. Eur Heart J. 2005; 26:2277-84. https://doi.org/10.1093/eurheartj/ehi406.

17. Januzzi JL, van Kimmenade R, Lainchbury J, BayesGenis A, Ordonez-Llanos J, Santalo-Bel M, Pinto YM, Richards M. NT-proBNP testing for diagnosis and short-term prognosis in acute destabilized heart failure: an international pooled analysis of 1256 patients: the International Collaborative of NT-proBNP Study. Eur Heart J. 2006; 27:330-7. https://doi.org/10.1093/eurheartj/ehi631.

18. Palladini G, Campana C, Klersy C, Balduini A, Vadacca G, Perfetti V, Perlini S, Obici L, Ascari E, d'Eril GM, Moratti R, Merlini G. Serum N-terminal pro-brain natriuretic peptide is a sensitive marker of myocardial dysfunction in $\mathrm{AL}$ amyloidosis. Circulation. 2003; 107:2440-5. https://doi. org/10.1161/01.Cir.0000068314.02595.B2.

19. Kumar S, Dispenzieri A, Lacy MQ, Hayman SR, Buadi FK, Colby C, Laumann K, Zeldenrust SR, Leung N, Dingli D, Greipp PR, Lust JA, Russell SJ, et al. Revised prognostic staging system for light chain amyloidosis incorporating cardiac biomarkers and serum free light chain measurements. J Clin Oncol. 2012; 30:989-95. https://doi. org/10.1200/jco.2011.38.5724.

20. McGrady M, Reid CM, Shiel L, Wolfe R, Boffa U, Liew D, Campbell DJ, Prior D, Krum H. N-terminal B-type natriuretic peptide and the association with left ventricular diastolic function in a population at high risk of incident heart failure: results of the SCReening Evaluationof the Evolution of NewHeart Failure Study (SCREEN-HF). Eur J Heart Fail. 2013; 15:573-80. https://doi.org/10.1093/eurjhf/hft001.

21. Zver S, Zadnik V, Cernelc P, Kozelj M. Cardiac toxicity of high-dose cyclophosphamide and melphalan in patients with multiple myeloma treated with tandem autologous hematopoietic stem cell transplantation. Int J Hematol. 2008; 88:227-36. https://doi.org/10.1007/s12185-008-0112-5.

22. Feliz V, Saiyad S, Ramarao SM, Khan H, Leonelli F, Guglin M. Melphalan-induced supraventricular tachycardia: incidence and risk factors. Clin Cardiol. 2011; 34:356-9. https://doi.org/10.1002/clc.20904.

23. Kumar SK, Rajkumar SV, Dispenzieri A, Lacy MQ, Hayman SR, Buadi FK, Zeldenrust SR, Dingli D, Russell SJ, Lust JA, Greipp PR, Kyle RA, Gertz MA. Improved survival in multiple myeloma and the impact of novel therapies. Blood. 2008; 111:2516-20. https://doi. org/10.1182/blood-2007-10-116129.

24. Willis MS, Patterson C. Proteotoxicity and cardiac dysfunction--Alzheimer's disease of the heart? N Engl J Med. 2013; 368:455-64. https://doi.org/10.1056/NEJMra1106180.

25. Wan SH, Vogel MW, Chen HH. Pre-clinical diastolic dysfunction. J Am Coll Cardiol. 2014; 63:407-16. https:// doi.org/10.1016/j.jacc.2013.10.063.

26. van Rhee F, Bolejack V, Hollmig K, Pineda-Roman M, Anaissie E, Epstein J, Shaughnessy JD Jr, Zangari M, Tricot G, Mohiuddin A, Alsayed Y, Woods G, Crowley J, et al. High serum-free light chain levels and their rapid reduction in response to therapy define an aggressive multiple myeloma subtype with poor prognosis. Blood. 2007; 110:827-32. https://doi.org/10.1182/blood-2007-01-067728.

27. Snozek CL, Katzmann JA, Kyle RA, Dispenzieri A, Larson DR, Therneau TM, Melton LJ 3rd, Kumar S, Greipp PR, Clark RJ, Rajkumar SV. Prognostic value of the serum free light chain ratio in newly diagnosed myeloma: proposed incorporation into the international staging system. Leukemia. 2008; 22:1933-7. https://doi. org/10.1038/leu.2008.171.

28. Zagouri F, Kastritis E, Zomas A, Terpos E, Katodritou E, Symeonidis A, Delimpasi S, Pouli A, Vassilakopoulos TP, Michalis E, Giannouli S, Kartasis Z, Christoforidou A, et al. Hypercalcemia remains an adverse prognostic factor 
for newly diagnosed multiple myeloma patients in the era of novel antimyeloma therapies. Eur J Haematol. 2017; 99:409-14. https://doi.org/10.1111/ejh.12923.

29. Raymond I, Groenning BA, Hildebrandt PR, Nilsson JC, Baumann M, Trawinski J, Pedersen F. The influence of age, sex and other variables on the plasma level of N-terminal pro brain natriuretic peptide in a large sample of the general population. Heart. 2003; 89:745-51.

30. Rajkumar SV, Dimopoulos MA, Palumbo A, Blade J, Merlini G, Mateos MV, Kumar S, Hillengass J, Kastritis E, Richardson P, Landgren O, Paiva B, Dispenzieri A, et al. International Myeloma Working Group updated criteria for the diagnosis of multiple myeloma. Lancet Oncol. 2014; 15:e538-48. https://doi.org/10.1016/ s1470-2045(14)70442-5.

31. Kumar S, Paiva B, Anderson KC, Durie B, Landgren O, Moreau P, Munshi N, Lonial S, Blade J, Mateos MV, Dimopoulos M, Kastritis E, Boccadoro M, et al. International Myeloma Working Group consensus criteria for response and minimal residual disease assessment in multiple myeloma. Lancet Oncol. 2016; 17:e328-e46. https://doi.org/10.1016/S1470-2045(16)30206-6.
32. Nagueh SF, Smiseth OA, Appleton CP, Byrd BF 3rd, Dokainish H, Edvardsen T, Flachskampf FA, Gillebert TC, Klein AL, Lancellotti P, Marino P, Oh JK, Popescu BA, et al. Recommendations for the Evaluation of Left Ventricular Diastolic Function by Echocardiography: An Update from the American Society of Echocardiography and the European Association of Cardiovascular Imaging. J Am Soc Echocardiogr. 2016; 29:277-314. https://doi. org/10.1016/j.echo.2016.01.011.

33. DeLong ER, DeLong DM, Clarke-Pearson DL. Comparing the areas under two or more correlated receiver operating characteristic curves: a nonparametric approach. Biometrics. 1988; 44:837-45.

34. Pencina MJ, D’Agostino RB Sr, Steyerberg EW. Extensions of net reclassification improvement calculations to measure usefulness of new biomarkers. Stat Med. 2011; 30:11-21. https://doi.org/10.1002/sim.4085.

35. Hosmer DW, Hosmer T, Le Cessie S, Lemeshow S. A comparison of goodness-of-fit tests for the logistic regression model. Stat Med. 1997; 16:965-80. 